\title{
DARBOUX INVARIANTS FOR PLANAR POLYNOMIAL DIFFERENTIAL SYSTEMS HAVING AN INVARIANT CONIC
}

\author{
JAUME LLIBRE ${ }^{1}$, MARCELO MESSIAS $^{2}$ AND ALISSON C. REINOL ${ }^{3}$
}

\begin{abstract}
We characterize all the planar polynomial differential systems with a unique invariant algebraic curve, which is a conic, and having a Darboux invariant.
\end{abstract}

\section{INTRODUCTION AND STATEMENT OF THE MAIN RESUltS}

Real planar polynomial differential systems appear in many branches of applied mathematics, physics and, in general, in applied sciences. For such differential systems the existence of a first integral determines completely their phase portrait. The first integrals depending on the time, i.e. on the independent variable of the differential system, are called invariants. A special class of invariants are the Darboux invariants. As we shall see the invariants instead of determining the phase portrait of the system, determine its $\alpha$ - and $\omega$-limit sets in the compactified polynomial differential system. That is, the Darboux invariants allow to describe the sets where all the orbits born or die.

In general it is a very difficult problem to recognize when a given polynomial differential system in the plane has or not a first integral or a Darboux invariant. The goal of this paper is to classify all polynomial differential systems in the plane $\mathbb{R}^{2}$ having a Darboux invariant and a unique invariant algebraic curve given by a conic.

Let $\mathbb{K}[x, y]$ be the ring of the polynomials in the variables $x$ and $y$ with coefficients in $\mathbb{K}$, where $\mathbb{K}$ is either $\mathbb{R}$ or $\mathbb{C}$. We consider the polynomial differential system in $\mathbb{R}^{2}$ defined by

$$
\dot{x}=P(x, y), \quad \dot{y}=Q(x, y),
$$

where $P, Q \in \mathbb{R}[x, y], P$ and $Q$ are relatively prime in $\mathbb{R}[x, y]$, and the dot denotes derivative with respect to the independent variable $t$ usually called the time.

2010 Mathematics Subject Classification. 34C05, 34C99.

Key words and phrases. Polynomial differential systems, invariant conics, Darboux integrability, Darboux invariant.

The first author is partially supported by the MINECO/FEDER grant MTM2008-03437, the Generalitat de Catalunya grant 2009SGR-410, ICREA Academia, and two grants FP7PEOPLE-2012-IRSES 316338 and 318999. The second author is supported by CNPq-Brazil grant 308315/2012-0 and by FAPESP grant 12/18413-7. The third author is supported by FAPESP grant 2013/01743-7. All the authors are supported by the Int. Coop. Proj. CAPES/MECDTQED II and PHB-2009-0025. 
We say that $m=\max \{\operatorname{deg} P, \operatorname{deg} Q\}$ is the degree of system (1). We associate to system (1) the vector field

$$
X=P(x, y) \frac{\partial}{\partial x}+Q(x, y) \frac{\partial}{\partial y} .
$$

We say that the polynomial differential system (1) is integrable on an open subset $U \subset \mathbb{R}^{2}$ if there exists a non-locally constant analytic function $H: U \rightarrow \mathbb{R}$, called a first integral of the system on $U$, which is constant on all solution curves $(x(t), y(t))$ of system (1) contained in $U$. Clearly $H$ is a first integral of system (1) if and only if $X H \equiv 0$ on $U$.

An invariant of system (1) on the open subset $U$ of $\mathbb{R}^{2}$ is a non-locally constant analytic function $I$ in the variables $x, y$ and $t$ such that $I$ is constant on all solution curves $(x(t), y(t))$ of system (1) contained in $U$, i.e.

$$
\frac{d I}{d t}=\frac{\partial I}{\partial x} P+\frac{\partial I}{\partial y} Q+\frac{\partial I}{\partial t}=0 .
$$

Obviously $I$ is a first integral of system (1) depending on the time $t$.

Let $f \in \mathbb{C}[x, y] \backslash\{0\}$. The algebraic curve $f(x, y)=0$ is an invariant algebraic curve of system (1) if for some polynomial $K \in \mathbb{C}[x, y]$ we have

$$
X f=P \frac{\partial f}{\partial x}+Q \frac{\partial f}{\partial y}=K f .
$$

The polynomial $K$ is called the cofactor of invariant algebraic curve $f=0$. Note that when $K=0$, then $f$ is a polynomial first integral. We remark that in the definition of invariant algebraic curve $f=0$ we always allow this curve to be complex, even in the case of a real polynomial system, due to the fact that sometimes for real polynomial systems the existence of a real first integral can be forced by the existence of complex invariant algebraic curves. For more details on invariant algebraic curves see [8].

Let $g, h \in \mathbb{C}[x, y] \backslash\{0\}$ and assume that $g$ and $h$ are relatively prime in the ring $\mathbb{C}[x, y]$ or that $h=1$. Then the function $\exp (g / h)$ is called an exponential factor of system (1) if for some polynomial $L \in \mathbb{C}[x, y]$ of degree at most $m-1$ we have that

$$
X(\exp (g / h))=L \exp (g / h) .
$$

We say that an invariant $I$ is a Darboux invariant of the vector field $X$ if it can be written as

$$
I(x, y, t)=f_{1}^{\lambda_{1}} \ldots f_{p}^{\lambda_{p}} F_{1}^{\mu_{1}} \ldots F_{q}^{\mu_{q}} e^{s t},
$$

where $f_{i}=0$ are invariant algebraic curves of $X$ for $i=1, \ldots, p ; F_{j}$ are exponential factors of $X$ for $j=1, \ldots, q ; \lambda_{i}, \mu_{j} \in \mathbb{C}$ and $s \in \mathbb{R} \backslash\{0\}$.

The search of first integrals is a classic tool in order to describe the phase portraits of a 2-dimensional differential system. As usual the phase portrait of a system is the decomposition of the domain of definition of this system as union of all its orbits. Every planar polynomial differential system in $\mathbb{R}^{2}$ can be analytically extended to infinity, in such a way that $\mathbb{R}^{2}$ is identified with the interior of a disc and its boundary $\mathbb{S}^{1}$ is identified with the infinity. This closed disc is called the Poincaré disc, for more details see Chapter 5 of [7]. The phase portrait of any planar polynomial differential system can be drawn on the Poincaré disc. 
Let $\phi_{p}(t)$ be the solution of system (1) passing through the point $p \in \mathbb{R}^{2}$, defined on its maximal interval $\left(\alpha_{p}, \omega_{p}\right)$ such that $\phi_{p}(0)=p$. If $\omega_{p}=\infty$ we define the set $\omega(p)=\left\{q \in \mathbb{R}^{2}\right.$ : there exist $\left\{t_{n}\right\}$ with $t_{n} \rightarrow \infty$ and $\phi\left(t_{n}\right) \rightarrow q$ when $\left.n \rightarrow \infty\right\}$.

In the same way, if $\alpha_{p}=-\infty$ we define the set

$\alpha(p)=\left\{q \in \mathbb{R}^{2}\right.$ : there exist $\left\{t_{n}\right\}$ with $t_{n} \rightarrow-\infty$ and $\phi\left(t_{n}\right) \rightarrow q$ when $\left.n \rightarrow \infty\right\}$.

The sets $\omega(p)$ and $\alpha(p)$ are called the $\omega$-limit set (or simply $\omega$-limit) and the $\alpha$-limit set (or $\alpha$-limit) of $p$, respectively.

The Darboux invariant provides information about the $\omega$ - and $\alpha$-limit sets of all orbits of system (1). More precisely, there is the following result proved in [10].

Proposition 1. Let $I(x, y, t)=f(x, y) e^{s t}$ be a Darboux invariant of system (1). Let $p \in \mathbb{R}^{2}$ and $\phi_{p}(t)$ the solution of system (1) with maximal interval $\left(\alpha_{p}, \omega_{p}\right)$ such that $\phi_{p}(0)=p$.

(a) If $\omega_{p}=\infty$ then $\omega(p) \subset \overline{\{f(x, y)=0\}}$ where the closure is taken inside the Poincaré disc.

(b) If $\alpha_{p}=-\infty$ then $\alpha(p) \subset \overline{\{f(x, y)=0\}}$ where the closure is taken inside the Poincaré disc.

The conics in $\mathbb{R}^{2}$ are classified as ellipses (E), complex ellipses (CE), hyperbolas $(\mathrm{H})$, parabolas $(\mathrm{P})$, two real straight lines intersecting at a point $(\mathrm{LV})$, two real and parallel straight lines (RPL), two complex and parallel straight lines (CPL), one double real straight line (DL), and two complex straight lines intersecting at a real point $p(\mathrm{p})$.

The characterization of all quadratic systems, i.e. system (1) with $m=2$, having two real or complex invariant straight lines taking into account their multiplicity was given in [2], and extensions to dimension 3 are given in [11].

Now we do the characterization of all polynomial differential systems in $\mathbb{R}^{2}$ having an invariant conic and a Darboux invariant.

Theorem 2 (LV). Every real polynomial differential system in $\mathbb{R}^{2}$ having a Darboux invariant and two real invariant straight lines intersecting at a point, after an affine change of coordinates, can be written as

$$
\dot{x}=x K_{1}(x, y), \quad \dot{y}=y\left(a+b K_{1}(x, y)\right),
$$

where $K_{1} \in \mathbb{R}[x, y], a, b \in \mathbb{R}$ and $a \neq 0$. Moreover its Darboux invariant is $I_{1}(x, y, t)=y /\left(x^{b} e^{a t}\right)$.

The polynomial differential systems in the plane having two real invariant straight lines intersecting at a point always can be written (as it is also showed in the proof of Theorem 2) into the form $\dot{x}=x g(x, y), \dot{y}=y h(x, y)$, which are the well known Lotka-Volterra systems. For this reason we denote by LV the polynomial differential systems having two real invariant straight lines intersecting in a point.

Theorem 2 for the particular case of quadratic systems was proved in [1].

Theorem 3 (RPL). Every real polynomial differential system in $\mathbb{R}^{2}$ having a Darboux invariant and two real and parallel invariant straight lines, after an affine change of coordinates and a rescaling of the time, can be written as

$$
\dot{x}=x^{2}-1, \quad \dot{y}=Q(x, y),
$$


where $Q \in \mathbb{R}[x, y]$. Moreover its Darboux invariant is $I_{2}(x, y, t)=\sqrt{\frac{x+1}{x-1}} e^{t}$.

Theorem 4 (CPL). Every real polynomial differential system in $\mathbb{R}^{2}$ having a Darboux invariant and two complex and parallel invariant straight lines, after an affine change of coordinates and a rescaling of the time, can be written as

$$
\dot{x}=x^{2}+1, \quad \dot{y}=Q(x, y),
$$

where $Q \in \mathbb{R}[x, y]$. Moreover its Darboux invariant is $I_{3}(x, y, t)=e^{(t+\arctan (1 / x))}$.

Theorem 5 (DL). Every real polynomial differential system in $\mathbb{R}^{2}$ having a Darboux invariant and one double real invariant straight line, after an affine change of coordinates and a rescaling of the time, can be written as

$$
\dot{x}=x^{2}, \quad \dot{y}=Q(x, y),
$$

where $Q \in \mathbb{R}[x, y]$. Moreover its Darboux invariant is $I_{4}(x, y, t)=e^{(t+1 / x)}$.

See the definition of a double real invariant straight line or an invariant straight line of multiplicity 2 in section 2 .

Theorem $6(\mathrm{p})$. Every real polynomial differential system in $\mathbb{R}^{2}$ having a Darboux invariant and two complex invariant straight lines intersecting at a real point, after an affine change of coordinates, can be written either as

$$
\dot{x}=(x-a y) A(x, y)-b y, \quad \dot{y}=(a x+y) A(x, y)+b x,
$$

where $A \in \mathbb{R}[x, y], a, b \in \mathbb{R}$, with $b \neq 0$, and its Darboux invariant is $I_{5}(x, y, t)=$ $\left(x^{2}+y^{2}\right)^{a} e^{-2 \arctan (y / x)+2 b t}$, or as

$$
\dot{x}=-y B(x, y)+c x, \quad \dot{y}=x B(x, y)+c y,
$$

where $B \in \mathbb{R}[x, y], c \in \mathbb{R} \backslash\{0\}$, and its Darboux invariant is $I_{6}(x, y, t)=\left(x^{2}+\right.$ $\left.y^{2}\right) e^{-2 c t}$.

Theorems 3, 4, 5 and 6 for the particular case of quadratic systems were proved in $[10]$.

Theorem $7(\mathrm{P})$. Every real polynomial differential system in $\mathbb{R}^{2}$ having a Darboux invariant and an invariant parabola, after an affine change of coordinates, can be written as

(8) $\dot{x}=\left(y-x^{2}\right) A(x, y)-C(x, y), \quad \dot{y}=\left(y-x^{2}\right)(b+2 x A(x, y))-2 x C(x, y)$,

where $b \in \mathbb{R} \backslash\{0\}$ and $A, C \in \mathbb{R}[x, y]$. Moreover its Darboux invariant is $I_{7}(x, y, t)=$ $e^{t} /\left(y-x^{2}\right)^{1 / b}$.

Theorem 8 (E, CE, H). Every real polynomial differential system in $\mathbb{R}^{2}$ having only one invariant algebraic curve given by either an ellipse, or a complex ellipse, or a hyperbola has no Darboux invariants.

This paper is organized as follows. In section 2 we present some results of the Darboux theory of integrability which we will use later on. In section 3 we prove Theorems 2 to 8 . 


\section{DARBOUX THEORY OF INTEGRABILITY}

In this section we introduce some results on the Darboux theory of integrability which shall be used in the next section to prove Theorems 2, 3, 4, 5, 6, 7 and 8 . This kind of integrability provides a link between the integrability of polynomial differential systems and their invariant algebraic curves. More details about this theory can be found in [4] and [7].

The following proposition is easy to prove.

Proposition 9. For the real polynomial differential system (1), $f=0$ is an invariant algebraic curve with cofactor $K$ if and only if $\bar{f}=0$ is an invariant algebraic curve with cofactor $\bar{K}$, where $\bar{f}$ and $\bar{K}$ denote the conjugates of $f$ and $K$, respectively.

Observe that if among the invariant algebraic curves of system (1) a complex conjugate pair $f=0$ and $\bar{f}=0$ occurs, then the Darboux invariant has a real factor of the form $f^{\lambda} \bar{f}^{\bar{\lambda}}$, which is the multi-valued real function

$$
\left[(\operatorname{Re}(f))^{2}+(\operatorname{Im}(f))^{2}\right]^{\operatorname{Re}(\lambda)} \exp (-2 \operatorname{Im}(\lambda) \arctan (\operatorname{Im}(f) / \operatorname{Re}(f))) .
$$

So if system (1) is real then the Darboux invariant is also real, independently of the fact of having complex invariant curves or complex exponential factors.

The next result is proved in Proposition 8.4 of [7].

Proposition 10. Suppose $f \in \mathbb{C}[x, y]$ and let $f=f_{1}^{n_{1}} \ldots f_{r}^{n_{r}}$ be its factorization into irreducible factors over $\mathbb{C}[x, y]$. Then for a polynomial differential system (1), $f=0$ is an invariant algebraic curve with cofactor $K_{f}$ if and only if $f_{i}=0$ is an invariant algebraic curve for each $i=1, \ldots, r$ with cofactor $K_{f_{i}}$. Moreover $K_{f}=n_{1} K_{f_{1}}+\ldots+n_{r} K_{f_{r}}$.

About exponential factors it is known the following result (for a proof see Proposition 8.6 of [7]).

Proposition 11. If $F=\exp (g / h)$ is an exponential factor for the polynomial differential system (1) and $h$ is not a constant, then $h=0$ is an invariant algebraic curve and $g$ satisfies the equation $X g=g K_{h}+h K_{F}$, where $K_{h}$ and $K_{F}$ are the cofactors of $h$ and $F$, respectively.

The next result and its proof can be found in [7] (see statement (vi) of Theorem 8.7) and it explains how to find Darboux invariants.

Proposition 12. Suppose that a polynomial differential system (1) of degree $m$ admits $p$ invariant algebraic curves $f_{i}=0$ with cofactors $K_{i}$ for $i=1, \ldots, p$, and $q$ exponential factors $F_{j}=\exp \left(g_{j} / h_{j}\right)$ with cofactors $L_{j}$ for $j=1, \ldots, q$. There exist $\lambda_{i}, \mu_{j} \in \mathbb{C}$ not all zero such that

$$
\sum_{p}^{i=1} \lambda_{i} K_{i}+\sum_{q}^{j=1} \mu_{j} L_{j}=-s,
$$

for some $s \in \mathbb{R} \backslash\{0\}$, if and only if the real (multi-valued) function

$$
f_{1}^{\lambda_{1}} \ldots f_{p}^{\lambda_{p}} F_{1}^{\mu_{1}} \ldots F_{q}^{\mu_{q}} e^{s t}
$$

is a Darboux invariant of system (1). 
The next theorem, which is due to Christopher [3] and was rediscovered by Zholadek [12], an algebraic proof of it can be found in [6], shows that for the integrability of a polynomial differential system (1) of degree $m$ we do not need many algebraic solutions when these solutions are in generic position. Then it is enough that the sum of their degrees be $m+1$.

Theorem 13 (Christopher-Zholadek Theorem). Let $f_{i}=0$ for $i=1, \ldots, p$ be $p$ irreducible algebraic curves in $\mathbb{C}^{2}$, and let $k=\sum_{i=1}^{q} \operatorname{deg} f_{i}$. We assume

(i) there are no points at which $f_{i}$ and its first derivatives all vanish,

(ii) the highest order terms of $f_{i}$ have no repeated factors,

(iii) no more than two curves meet at any point in the finite plane and are not tangent at these points,

(iv) no two curves have a common factor in their highest order terms,

then any polynomial vector field $X$ of degree $m$ tangent to all $f_{i}=0$ is of the form described bellow.

(a) If $m>k-1$ then

$$
X=Y\left(\prod_{i=1}^{p} f_{i}\right)+\sum_{i=1}^{p} h_{i}\left(\prod_{\substack{j=1, j \neq i}}^{p} f_{j}\right) X_{f_{i}},
$$

where $X_{f_{i}}=\left(-\partial f_{i} / \partial y, \partial f_{i} / \partial x\right)$ is a Hamiltonian vector field, the $h_{i}$ are polynomials of degree $\leq m-k+1$ and $Y$ is a polynomial vector field of degree $\leq m-k$.

(b) If $m=k-1$ then

$$
X=\sum_{i=1}^{p} \alpha_{i}\left(\prod_{\substack{j=1, j \neq i}}^{p} f_{j}\right) X_{f_{i}}
$$

with $\alpha_{i} \in \mathbb{C}$. In this case, a Darboux first integral exists.

(c) If $m<k-1$ then $X \equiv 0$.

It is known that if a planar polynomial differential system (1) has the invariant straight line $a+b x+c y=0$ then $a+b x+c y$ divides the polynomial

$$
R(x, y)=\left|\begin{array}{ccc}
1 & x & y \\
0 & P & Q \\
0 & P P_{x}+Q P_{y} & P Q_{x}+Q Q_{y}
\end{array}\right| .
$$

Moreover, if $(a+b x+c y)^{k} \mid R(x, y)$ and $(a+b x+c y)^{k+1} \nmid R(x, y)$ then we say that the invariant straight line $a+b x+c y=0$ has multiplicity $k$. For more details on the multiplicity of invariant straight lines see [5] and [9].

The proof of the next result can be found in [6].

Lemma 14. Assume that the polynomial differential system (1) has an invariant algebraic curve $f=0$ and that $f$ satisfies condition (i) of Theorem 13. If $\left(f_{x}, f_{y}\right)=$ 1 , then system (1) has the following normal form

$$
\dot{x}=A f-C f_{y}, \quad \dot{y}=B f+C f_{x}
$$

where $A, B, C \in \mathbb{R}[x, y], f_{x}=\partial f / \partial x, f_{y}=\partial f / \partial y$. Here $\left(f_{x}, f_{y}\right)=1$ means that the greatest common divisor between $f_{x}$ and $f_{y}$ is 1 . 


\section{Proof of the theorems}

Now we will prove Theorems $2,3,4,5,6,7$ and 8 , which were stated in section 1.

Proof of Theorem $2(L V)$. Suppose that the polynomial differential system (1) has two real invariant straight lines intersecting at a point. Then, after an affine change of coordinates, we can assume that $f_{1}=x=0$ and $f_{2}=y=0$ are the invariant straight lines of system (1) intersecting at the origin with cofactors $K_{1}$ and $K_{2}$, respectively. So we have that

$$
P \frac{\partial f_{1}}{\partial x}+Q \frac{\partial f_{1}}{\partial y}=K_{1} f_{1} \quad \Rightarrow \quad P=K_{1} x .
$$

Analogously,

$$
P \frac{\partial f_{2}}{\partial x}+Q \frac{\partial f_{2}}{\partial y}=K_{2} f_{2} \quad \Rightarrow \quad Q=K_{2} y
$$

Therefore, we can write system (1) as

$$
\dot{x}=x K_{1}(x, y), \quad \dot{y}=y K_{2}(x, y),
$$

where $K_{1}, K_{2} \in \mathbb{R}[x, y]$.

Now we suppose that system (12) has a Darboux invariant. By Proposition 12 there exist $\mu, \lambda \in \mathbb{C}$ not all zero such that from equation (10) we have $\mu K_{1}+\lambda K_{2}=$ $-s$, where $s \in \mathbb{R} \backslash\{0\}$. In particular, without loss of generality we can consider $\mu, \lambda \in \mathbb{R}$ and $\lambda \neq 0$. Then

$$
K_{2}=-\frac{s}{\lambda}-\frac{\mu}{\lambda} K_{1}
$$

Therefore, from system (12) we get the following system

$$
\dot{x}=x K_{1}(x, y), \quad \dot{y}=-\frac{1}{\lambda} y\left(s+\mu K_{1}(x, y)\right) .
$$

So taking $a=-s / \lambda$ and $b=-\mu / \lambda$ we obtain system (2). Moreover, it follows directly from Proposition 12 that the Darboux invariant of system (2) is given by $I_{1}(x, y, t)=y /\left(x^{b} e^{a t}\right)$.

Proof of Theorem 3 (RPL). Suppose that the polynomial differential system (1) has two real and parallel invariant straight lines. Then, after an affine change of coordinates, we can take $f_{1}=x-1=0$ and $f_{2}=x+1=0$ as the invariant parallel straight lines of system (1) with cofactors $K_{1}$ and $K_{2}$, respectively. So we have that

$$
P \frac{\partial f_{1}}{\partial x}+Q \frac{\partial f_{1}}{\partial y}=K_{1} f_{1} \quad \Rightarrow \quad P=K_{1}(x-1) .
$$

Analogously,

$$
P \frac{\partial f_{2}}{\partial x}+Q \frac{\partial f_{2}}{\partial y}=K_{2} f_{2} \quad \Rightarrow \quad P=K_{2}(x+1) .
$$

Then $P(x, y)=\left(x^{2}-1\right) g(x, y)$, with $g \in \mathbb{R}[x, y]$. Therefore, we can write system (1) as

$$
\dot{x}=\left(x^{2}-1\right) g(x, y), \quad \dot{y}=Q(x, y) .
$$

Now we suppose that system (13) has a Darboux invariant. By Proposition 12 there exist $\mu, \lambda \in \mathbb{C}$ not all zero such that they satisfy (10). Since $K_{1}=(x+1) g(x, y)$ 
and $K_{2}=(x-1) g(x, y)$ are the cofactors of $f_{1}=0$ and $f_{2}=0$ respectively, then from (10) we have

(14) $\mu(x+1) g(x, y)+\lambda(x-1) g(x, y)=-s \quad \Rightarrow \quad[\mu(x+1)+\lambda(x-1)] g(x, y)=-s$.

Hence $g(x, y)=a \in \mathbb{R} \backslash\{0\}$, because otherwise $s=0$, which is a contradiction with the fact that system (13) has a Darboux invariant. Then we can write system (13) as

$$
\dot{x}=a\left(x^{2}-1\right), \quad \dot{y}=Q(x, y) .
$$

We obtain system (3) doing the rescaling $T=a t$, where $T$ is the new time. So we can take $g(x, y)=1$ in equation (14) and then we have that $\mu+\lambda=0$ and $\mu-\lambda=-s$. Hence $\lambda=-\mu=s / 2$. Then choosing $s=1$ it follows directly from Proposition 12 that the Darboux invariant of system (3) is given by $I_{2}(x, y, t)=\sqrt{\frac{x+1}{x-1}} e^{t}$.

Proof of Theorem $4(C P L)$. Suppose that the polynomial differential system (1) has two complex and parallel invariant straight lines. Then, after an affine change of coordinates, we can take $f_{1}=x-i=0$ and $f_{2}=x+i=0$ as the invariant parallel straight lines of system (1) with cofactors $K_{1}$ and $K_{2}$, respectively. Using the same arguments of the proof of the previous theorem we can assume $P(x, y)=$ $\left(x^{2}+1\right) g(x, y)$, with $g \in \mathbb{R}[x, y]$. Therefore, we can write system (1) as

$$
\dot{x}=\left(x^{2}+1\right) g(x, y), \quad \dot{y}=Q(x, y),
$$

where $Q(x, y) \in \mathbb{R}[x, y]$.

Now we suppose that system (15) has a Darboux invariant. By Proposition 12 there exist $\mu, \lambda \in \mathbb{C}$ not all zero such that they satisfy (10). Since $K_{1}=(x+i) g(x, y)$ and $K_{2}=\bar{K}_{1}$ are the cofactors of $f_{1}=0$ and $f_{2}=0$ respectively, then from (10) we have

$$
[\mu(x+i)+\lambda(x-i)] g(x, y)=-s .
$$

As before we have $g(x, y)=a \in \mathbb{R} \backslash\{0\}$. Then we can write system (15) as

$$
\dot{x}=a\left(x^{2}+1\right), \quad \dot{y}=Q(x, y) .
$$

We obtain system (4) doing the rescaling $T=a t$, where $T$ is the new time.

Now we consider $\mu=\mu_{1}+i \mu_{2}$, with $\mu_{1}, \mu_{2} \in \mathbb{R}$. From equation (16) note that $\lambda=\bar{\mu}$. So we obtain $2 a\left(\mu_{1} x-\mu_{2}\right)=-s$, and consequently $\mu_{1}=0$ and $\mu_{2}=s / 2$. It follows from Proposition 12 that the Darboux invariant of system (4) is given by $I_{3}(x, y, t)=(x-i)^{(i s / 2)}(x+i)^{(-i s / 2)} e^{s t}$. Then using (9) we can replace $f_{1}^{\mu} \bar{f}_{1}^{\bar{\mu}}$ and we obtain $I_{3}(x, y, t)=e^{(t+\arctan (1 / x))}$.

Proof of Theorem $5(D L)$. Suppose that the polynomial differential system (1) has one double real invariant straight line. Then, after an affine change of coordinates, we can consider $f=x=0$ as the double real invariant straight line of system (1). Given a small perturbation of system (1), the invariant straight line $x=0$ bifurcates in two real and parallel straight lines $f_{1}=x-\varepsilon=0$ and $f_{2}=x+\varepsilon=0$. Other possibility is that $x=0$ bifurcates in two real invariant straight lines intersecting at a point, but in this case, after doing some computations, we obtain that the polynomials $P$ and $Q$ of system (1) are not relatively prime. So, from the proof of Theorem 3 (RPL), we can write system (1) as

$$
\dot{x}=\left(x^{2}-\varepsilon^{2}\right) g(x, y), \quad \dot{y}=Q(x, y),
$$


where $g \in \mathbb{R}[x, y]$. Then making $\varepsilon \rightarrow 0$ we can write system (17) as

$$
\dot{x}=x^{2} g(x, y), \quad \dot{y}=Q(x, y),
$$

and note that $f=x=0$ is a double real invariant straight line of system (18).

Note that $f=x=0$ has cofactor $K=x g(x, y)$ and, by Proposition 11, we have that $F=e^{1 / x}$ is an exponential factor of system (18) with cofactor $L=-g(x, y)$. Suppose that system (18) has a Darboux invariant. So, by Proposition 12 there exist $\mu, \lambda \in \mathbb{C}$ not all zero such that

$$
\mu K+\lambda L=-s \quad \Rightarrow \quad(\mu x-\lambda) g(x, y)=-s,
$$

where $s \in \mathbb{R} \backslash\{0\}$. Using the same arguments of the proof of the previous theorems we can take $g(x, y)=a \in \mathbb{R} \backslash\{0\}$. Then we can write system (18) as

$$
\dot{x}=a x^{2}, \quad \dot{y}=Q(x, y) .
$$

We obtain system (5) doing the rescaling $T=a t$, where $T$ is the new time. So we can take $g(x, y)=1$ in equation (19). Then $\mu=0$ and $\lambda=s$. Hence it follows directly from Proposition 12 that the Darboux invariant of system (5) is given by $I_{4}(x, y, t)=e^{(t+1 / x)}$.

Proof of Theorem 6 (p). Suppose that the polynomial differential system (1) has two complex invariant straight lines intersecting at a real point. Then, after an affine change of coordinates, we can take $f_{1}=x+i y=0$ and $f_{2}=x-i y=0$ as the invariant straight lines of system (1) with cofactors $K_{1}$ and $K_{2}$, respectively. We denote the vector field of degree $m$ associated with system (1) by $X=(P, Q)$. According to Theorem 13, we can write

$$
X=f_{1} f_{2} Y+h_{1} f_{2} X_{f_{1}}+h_{2} f_{1} X_{f_{2}},
$$

where $X_{f_{i}}=\left(-\partial f_{i} / \partial y, \partial f_{i} / \partial x\right)$ is a Hamiltonian vector field, $h_{1}$ and $h_{2}$ are polynomials of degree $\leq m-1$, and $Y=\left(P_{1}, Q_{1}\right)$ is a polynomial vector field of degree $\leq m-2$. So $(P, Q)=f_{1} f_{2}\left(P_{1}, Q_{1}\right)+h_{1} f_{2}(-i, 1)+h_{2} f_{1}(i, 1)$ and consequently

$$
\begin{aligned}
& P=\left(x^{2}+y^{2}\right) P_{1}-\left(h_{1}+h_{2}\right) y+i\left(h_{2}-h_{1}\right) x, \\
& Q=\left(x^{2}+y^{2}\right) Q_{1}+\left(h_{1}+h_{2}\right) x+i\left(h_{2}-h_{1}\right) y .
\end{aligned}
$$

Let $h_{1}(x, y)=m_{1}(x, y)+i n_{1}(x, y)$ and $h_{2}(x, y)=m_{2}(x, y)+i n_{2}(x, y)$, where $m_{1}, m_{2}, n_{1}, n_{2} \in \mathbb{R}[x, y]$. Then we have that

$$
\begin{aligned}
& P=\left(x^{2}+y^{2}\right) P_{1}-y\left(\left(m_{1}+m_{2}\right)+i\left(n_{1}+n_{2}\right)\right)+x\left(\left(n_{1}-n_{2}\right)+\left(m_{2}-m_{1}\right) i\right), \\
& Q=\left(x^{2}+y^{2}\right) Q_{1}+x\left(\left(m_{1}+m_{2}\right)+i\left(n_{1}+n_{2}\right)\right)+y\left(\left(n_{1}-n_{2}\right)+\left(m_{2}-m_{1}\right) i\right) .
\end{aligned}
$$

So $n_{1}=-n_{2}$ and $m_{1}=m_{2}$. Therefore, we can write system (1) as

$$
\begin{aligned}
& \dot{x}=\left(x^{2}+y^{2}\right) P_{1}(x, y)-2 y m_{1}(x, y)+2 x n_{1}(x, y), \\
& \dot{y}=\left(x^{2}+y^{2}\right) Q_{1}(x, y)+2 x m_{1}(x, y)+2 y n_{1}(x, y) .
\end{aligned}
$$

Now we consider $m_{1}(x, y)=M(x, y)+m$ and $n_{1}(x, y)=N(x, y)+n$, with $M(0,0)=N(0,0)=0$. We can rewrite system (20) into the form

$$
\begin{aligned}
& \dot{x}=x \hat{A}(x, y)-y \hat{B}(x, y)+2(n x-m y), \\
& \dot{y}=y \hat{A}(x, y)+x \hat{B}(x, y)+2(n y+m x),
\end{aligned}
$$

where $\hat{A}(x, y)=x P_{1}(x, y)+y Q_{1}(x, y)+2 N(x, y)$ and $\hat{B}(x, y)=x Q_{1}(x, y)-$ $y P_{1}(x, y)+2 M(x, y)$. We suppose that system (21) has a Darboux invariant. By 
Proposition 12 there exist $\mu, \lambda \in \mathbb{C}$ not all zero such that they satisfy (10). We can show that $K_{1}=(x-i y)\left(P_{1}+i Q_{1}\right)+2 i(M+m)+2(N+n)$ and $K_{2}=\bar{K}_{1}$ are the cofactors of $f_{1}=0$ and $f_{2}=0$ respectively. Then from equation (10) we have

$$
(\mu+\lambda)\left(x P_{1}+y Q_{1}+2(N+n)\right)+(\mu-\lambda) i\left(x Q_{1}-y P_{1}+2(M+m)\right)=-s,
$$
i.e.

$$
(\mu+\lambda)(\hat{A}+2 n))+(\mu-\lambda) i(\hat{B}+2 m))=-s,
$$

So $\lambda=\bar{\mu}$. We take $\mu=\mu_{1}+i \mu_{2}$, where $\mu_{1}, \mu_{2} \in \mathbb{R}$. Then from equation (22) we obtain

$$
2 \mu_{1}(\hat{A}+2 n)-2 \mu_{2}(\hat{B}+2 m)=-s,
$$

which provides the following linear system

$$
\begin{aligned}
& \mu_{1} \hat{A}(x, y)-\mu_{2} \hat{B}(x, y)=0, \\
& \mu_{1} n-\mu_{2} m=-\frac{s}{4},
\end{aligned}
$$

in the variables $\mu_{1}$ and $\mu_{2}$.

We first consider $\mu_{2} \neq 0$. Then from equation (23) we have that $\hat{B}(x, y)=$ $\left(\mu_{1} / \mu_{2}\right) \hat{A}(x, y)$ and $m=s /\left(4 \mu_{2}\right)+n \mu_{1} / \mu_{2}$. We obtain system (6) replacing $\hat{B}$ and $m$ in system $(21)$ and taking $A(x, y)=\hat{A}(x, y)+2 n, a=\mu_{1} / \mu_{2}$ and $b=s /\left(2 \mu_{2}\right)$. Moreover from Proposition 12 and equation (9) it follows that the Darboux invariant of system $(6)$ is $I_{5}(x, y, t)=\left(x^{2}+y^{2}\right)^{a} e^{-2 \arctan (y / x)+2 b t}$.

Now we consider $\mu_{2}=0$. Obviously $\mu_{1} \neq 0$. So from equation (23) we have that $\hat{A}(x, y)=0$ and $n=-s /\left(4 \mu_{1}\right)$. We obtain system (7) replacing $\hat{A}$ and $n$ in system $(21)$ and taking $B(x, y)=\hat{B}(x, y)+2 m$ and $c=-s /\left(2 \mu_{1}\right)$. Moreover from Proposition 12 it follows that Darboux invariant of system $(7)$ is $I_{6}(x, y, t)=$ $\left(x^{2}+y^{2}\right) e^{-2 c t}$.

Proof of Theorem $7(P)$. Suppose that the polynomial differential system (1) has an invariant parabola. Then, after an affine change of coordinates, we can assume that $f=y-x^{2}=0$ is the invariant parabola of system (1) with cofactor $K$. Using Lemma 14 we can write system (1) as

$$
\dot{x}=\left(y-x^{2}\right) A(x, y)-C(x, y), \quad \dot{y}=\left(y-x^{2}\right) B(x, y)-2 x C(x, y),
$$

where $A, B, C \in \mathbb{R}[x, y]$.

Now we suppose that system (24) has a Darboux invariant. Then from Proposition 12 there exists $\mu \in \mathbb{R} \backslash\{0\}$ such that $\mu K=-s$, where $s \in \mathbb{R} \backslash\{0\}$ and $K=B(x, y)-2 x A(x, y)$. Let $B(x, y)=b+\hat{B}(x, y)$, with $\hat{B}(0,0)=0$. So $b=-s / \mu \neq 0$ and $\hat{B}(x, y)=2 x A(x, y)$. Replacing $B(x, y)$ in system (24) we obtain system (8). We get from Proposition 12 and choosing $s=1$ that the Darboux invariant of system $(8)$ is $I_{7}(x, y, t)=e^{t} /\left(y-x^{2}\right)^{1 / b}$.

Proof of Theorem 8 (E, CE, H). Suppose that the polynomial differential system (1) has an invariant ellipse. Then, after an affine change of coordinates, we can assume that $f=x^{2}+y^{2}-1=0$ is the invariant ellipse of system (1) with cofactor $K$. Using Lemma 14 we can write system (1) as

(25) $\dot{x}=\left(x^{2}+y^{2}-1\right) A(x, y)-2 y C(x, y), \quad \dot{y}=\left(x^{2}+y^{2}-1\right) B(x, y)+2 x C(x, y)$, where $A, B, C \in \mathbb{R}[x, y]$. 
It is easy to show that the cofactor of $f=0$ is $K=2(A x+B y)$. Then equation (10) of Proposition 12 becomes $\mu K=-s$, but since the polynomial $\mu K$ has no independent term the equation never holds. Therefore, by Proposition 12 it follows that system (25) does not have Darboux invariants.

Note that if the polynomial differential system (1) has either an invariant complex ellipse or an invariant hyperbola then, after an affine change of coordinates, we can consider $x^{2}+y^{2}+1=0$ and $x^{2}-y^{2}-1=0$ as the invariant complex ellipse and invariant hyperbola respectively. Analogously to the case of invariant ellipse we can prove that differential polynomial systems in $\mathbb{R}^{2}$ with these invariant conics do not have Darboux invariants.

\section{REFERENCES}

[1] Y. Bolaños, J. Llibre and C. Valls, Phase portraits of quadratic Lotka-Volterra systems with a Darboux invariant in the Poincaré disc, preprint, 2013.

[2] L. Cairó And J. Llibre, Darbouxian first integrals and invariants for real quadratic systems having an invariant conic, J. Phys. A: Math. Gen. 35 (2002), 589-608.

[3] C. Christopher, Invariant algebraic curves and conditions for a center, Proc. R. Soc. Edin. 124 A (1994), 1209-1229.

[4] C. Christopher and C. Li, Limit Cycles of Differential Equations, Birkhauser-Verlag, Basel, 2007.

[5] C. Christopher, J. Llibre and J.V. Pereira, Multiplicity of invariant algebraic curves in polynomial vector fields, Pacific J. Math. 229 (2007), 63-117.

[6] C. Christopher, J. Llibre, C. Pantazi and X. Zhang, Darboux integrability and invariant algebraic curves for planar polynomial systems, J. Phys. A: Math Gen. 35 (2002), 2457-2476.

[7] F. Dumortier, J. Llibre And J.C. Artés, Qualitative Theory of Planar Differential Systems, Springer-Verlag, New York, 2006.

[8] A. Ferragut, Some new results on Darboux integrable differential systems, J. Math. Anal. Appl. 394 (2012), 416-424.

[9] J. Llibre ANd J.C. MedRado, On the invariant hiperplanes for d-dimensional polynomial vector fields, J. Phys. A: Math Gen. 40 (2007), 8385-8391.

[10] J. Llibre AND R.D.S. OliveIRA, Quadratic systems with invariant straight lines with total multiplicity two having Darboux invariants, preprint, 2013.

[11] J.Llibre And C. Valls, Polynomial, rational and analytic first integrals for a family of 3-dimensional Lotka-Volterra systems, Z. Angew. Math. Phys. 62 (2011), 761-777.

[12] H. ZholadeK, On algebraic solutions of algebraic Pfaff equations, Studia Math. 114 (1995) $117-126$.

1 Departament de Matemàtiques, Universitat Autònoma de Barcelona, 08193 Bellaterra, Barcelona, Catalonia, Spain.

E-mail address: jllibre@mat.uab.cat

2,3 Departamento de Matemática e ComputaÇão - FCt-Unesp, Presidente Prudente, São Paulo, Brazil.

E-mail address: marcelo@fct.unesp.br

E-mail address: alisson_carv@hotmail.com 